have been nearly or quite equaled by prevention of phylloxera and studies on diseases of silkworms.

The real humanitarian battle-field, however, only commenced with his discoveries in regard to anthrax and the bacillus of malignant edema, hog erysipelas, chicken cholera and hydrophobia. Chicken cholera was especially interesting because, in studying this, he found that old cultures gave a mild disease on inoculation, which conferred immunity on the animal inoculated against the virulent disease, and thus was the theory of attenuated virus launched. By cultivating anthrax at a temperature of $107 \mathrm{~F}$. for eight days, spore fermentation was prevented and virus attenuated. From the decisive test of fifty inoculated sheep at Pouilly-le-Fort in 1881 until 1894 there were nearly four million sheep inoculated in France, with a mortality of only 1 per cent.; one-half a million cattle with less than 3 per cent. mortality. Pasteur's most popular discovery was the attenuation of the virus of hydrophobia. This dreadful disease, so frequent in France, appealed to the imagination of all, and the astonishing statistics published in 1894, of 20,000 patients treated with a mortality of only 5 per 1000 has conquered even the German prejudice.

But why try to enumerate the benefits of Pasteur's works? It can never be done. His influence and spirt of research have fallen like a mantle on his disciples, Roux, Duclaux, Metschnikoff, Borel, Martin, Calmette, and the natural respect among the French for their Maitres has in this case become a "cult" from whose chief temple high priests of science have gone forth to establish branch Pasteur institutes in India, China, Japan, Africa, Russia, and the United States. When the plague threatened Europe at Oporto,it was the Pasteur Institute which sent Calmette and Salimbeni, to whose efforts are due the splendid results of reducing the mortality from 62 per cent. in untreated to 14 per cent in those treated by pest antitoxin.

We visited the rest of the building, ascending to the large, handsome, well lighted library, with the current medical literature of the world as well as a mosts complete medical library, especially well supplied in works on bacteriology and pathology. Passing through the corridor to the rear building we turn to the right into the large laboratory for class instruction. Two courses of two months each are given here in microbiology-commencing in November and February. The cost is but nominal, nearly all material being furnished free. At no place in Paris are foreign medical men so well treated as at Pasteur's Institute, and while the French students usually have to inscribe a year in advance, foreigners are given places at once.

There is a probability of a course being given during the summer, especially for foreigners. M. Roux, whose modesty and enthusiasm for science make him a warm favorite wilh the students, has been in poor health for some time, and his course is given by Metschnikoff and Borel, with the collaboration of such specialists as Calmette on pests, Nocard on glanders, Martin on diphtheria, Vaillard on tetanus, Sabouraud on tricoph ytons, Morox on gonococci, Laveran on malaria, and others. The lectures are given from $1: 30$ to 3 p.m., after which there is practical work in all the processes used in bacteriology. Students who wish to follow some special line of work are allowed to continue after the termination of the spring course. If a worker already known for his researches desires the facilities of the Institute, the courtesies of Metschinkof"s laboratory on the floor above are usually extended.

Next to the general laboratory are the private laboratories of Roux and Borel, and returning to the main corridor we cross over into the domain of Duclaux and his corps of workers. Here is a most completely equipped chemical laboratory, where the successor of Pasteur as director of the Institute, reconciles into perfect harmony the sciences of bacteriology and chemistry. As professor in the Sorbonne he gives a course of lectures on biologic chemistry in his special amphitheater. As author of the most extensive work on bacteriology, not yet finished, as well as director of "Les Annales d l'Institut Pasteur," which was founded under the patronage of Pasteur in 1887 , and which contains in detail the unequaled work of the Institute for the past thirteen years, Duclaux is one of the hardest worked savants in Paris.

Ascending to the third floor, we find mostly private labora tories. Here are such men as Morox, Wasserman, and Christsnas, whose work on the gonococcus is well known, Marmorek, known for his antistreptococcic serum and others.

Descending to the first floor, to the right are the micro. photographic rooms, aquarium, and glass-blowers' room.

To better control its results the Institute tries to have every thing made on the spot. Hence they manufacture all the glassware used in cultures, peptones, etc. The librarian also fills the functions of lithographer for "Les Annales."

Crossing over to the western wing, we enter the service of the "rage" or hydrophobia, Here is a large waiting-room, to which, every day at 10 a.m., about fifty patients come for treatment. They pass first into the registry-room, where complete records of cases can be kept. Cases are eategoried under three groups: 1. Those where "rage" is demonstrated to exist in the animal inflicting the wound, as shown by inoculation into the brain of a rabbit, with resulting paraplegia and death in eight days. 2. Those with a veterinary surgeon's certificate. 3. Those with no scientific proof of the existence of rabies in the suspected animal. In the next room the inoculations are made. All necessary antiseptic precautions are used, and there are never any unpleasant symptoms following injections, which are commenced in a new case with triturations of the rabbit's spinal cord, which has been attenuated for fourteen days, increasing the intensity of the dose up to eight days' attenuation, then recommencing.

In the next room the rabbits are inoculated by trephining the skull and introducing the trituration of the spinal cord of the rabbit that died the day before. A rabbit has died of hydrophobia each day for twenty years. This maintains the culture in all its virulence. For the attentuation, acting on the same ideas as with chicken cholera, i. e., cultures in becoming old lose virulence, the removed cord is suspended in a sterile jar containing caustic postash in the bottom. This jar is placed in one of a row of compartments with wire gauze doors, in a dark room, kept at a uniform temperature of $23 \mathrm{C}$. At the end of fourteen days the cord loses its toxic properties. A control tube of bouillon into which a fragment of the cord was placed on removal from the rabbit indicates freedom from contamination. This must remain clear, otherwise the cord is condemned and not used. The rabbits which have been inoculated are kept in very hygienic cases which can be flushed and food introduced without disturbing the animals. Several outhouses contain guinea-pigs, rabbits, chickens, geese, ducks, pigeons, mice and rats for use in the laboratory. A separate house contains the cages of animals inoculated and under observation.

The farm adjunct at Garches, near St. Cloud, has 200 horses which furnish the antitoxins of diphtheria, tetanus and pest. A. D. Newborn, M.D.

\section{Bacteriology and Pathology Society.}

$$
\text { April 12, } 1900
$$

To the Editor: The undersigned believe that the time has come for the formation of a national society, devoted to the advancement of the interests of the medical sciences, as distinct from the clinical side of medicine. The proposed society will be specially concerned with pathology and bacteriology, and a meeting for discussion of its plan and scope will be held in Washington, D. C., on the afternoon of Monday, April 30, at 2:30 p.m., in the Arlington Hotel. The general plan of the Society, so far as yet suggested, is:

1. Membership is limited to those who have already accomplished creditable work in research in pathology or bacteriology. 2. Meetings to be held annually in some important center, as may be determined (in May or June). 3. Members of the society to see that the work done by them, or under their control, is represented at the meetings of the society.

\section{Very truly yours,}

Wm. T. Councilman.

HAROLD C. ERNST.

Simon Flexiner.

LUDVIG HeKTOEN.

WM. T. HoWARD, JR.

W. H. PABK.

Theobald Smith. 